\title{
MIXED BOUNDARY VALUE PROBLEMS FOR NONSTRICT HYPERBOLIC EQUATIONS
}

\author{
BY RICHARD BEALS ${ }^{1}$
}

\author{
Communicated by Felix Browder, December 29, 1971
}

The Cauchy problem for a class of nonstrict hyperbolic equations has been studied by Ohya and Leray [3], [5]. Recently Chazarain [2] has considered certain mixed problems for some fourth order nonstrict equations in the context of distribution semigroups. In this note we deal with mixed problems for a general class of (possibly) nonstrict equations of higher order, including those in [2]. The class of hyperbolic operators does not contain nor is it contained in those of [3], [5]. We do not require Gevrey class coefficients, nor that the principal part has real characteristics of constant multiplicity and therefore (see [4]) is a product of powers of strictly hyperbolic operators. We do, however, require the operators to be elliptic in the space variables alone and that the coefficients be independent of time. In this case recent results on the abstract Cauchy problem [1] can be applied. For simplicity we consider a single equation with smooth coefficients on a bounded region in euclidean space. Proofs will appear elsewhere.

Let $\Omega \subset \boldsymbol{R}^{n}, n \geqq 2$, be a bounded open set with boundary $\partial \Omega$ a regularly imbedded $C^{\infty}$ submanifold of $\boldsymbol{R}^{n}$. Let $a(y ; \xi, \lambda)$ be the polynomial

$$
\sum_{|\alpha|+j \leqq p} a_{\alpha j}(y) \xi^{\alpha} \lambda^{j}, \quad y \in \bar{\Omega}, \xi \in C^{n}, \lambda \in C .
$$

Let $a_{0}(y ; \xi, \lambda)$ be the principal part, the terms of total degree $p$. We assume that, for $y \in \bar{\Omega}, \xi \in \boldsymbol{R}^{n}$, the roots of $a_{0}(y ; \xi, \cdot)$ are all real and nonzero. This implies $p=2 r$ is even. Let $b_{k}(y ; \xi, \lambda)$ be polynomials of order $p_{k}<p$, $k=1,2, \ldots, r$, with principal part $b_{k 0}(y ; \xi, \lambda), y \in \partial \Omega, \xi \in C^{p}, \lambda \in C$. We assume the coefficients of these polynomials are $C^{\infty}$. We also assume the appropriate version of the "root covering condition" familiar from elliptic boundary value problems. Specifically, for $y \in \partial \Omega$, let $v=v(y)$ be the unit vector in $\boldsymbol{R}^{n}$ in the direction of the inner normal to $\partial \Omega$ at $y$, and let $W=W(y)$ be the subspace of $\boldsymbol{R}^{n}$ orthogonal to $v$. The assumptions imply that for $\xi \in W$ and $\operatorname{Re} \lambda>0$, or $\xi \in W \backslash(0)$ and $\lambda=0, a_{0}(y ; \mu \nu+i \xi, \lambda)$ has precisely $r$ roots $\mu_{1}(\xi, \lambda), \ldots, \mu_{r}(\xi, \lambda)$ with negative real part. Let

$$
q(\mu ; \xi, \lambda)=\Pi\left(\mu-\mu_{j}(\xi, \lambda)\right) .
$$

AMS 1970 subject classifications. Primary 35L35.

Key words and phrases. Mixed problems, initial boundary value problems, hyperbolic equations, multiple characteristics.

1 This research was supported in part by NSF Grant GP-28148. 
Then we assume that for each such $\xi, \lambda$, the polynomials

$$
b_{10}(y ; \mu \nu+i \xi, \lambda), \ldots, b_{r 0}(y ; \mu \nu+i \xi, \lambda)
$$

are linearly independent modulo $q(\mu ; \xi, \lambda)$ as polynomials in $\mu$.

We let the corresponding differential operators be

$$
\begin{aligned}
A u(y, t) & =a\left(y ; D_{y}, D_{t}\right) u(y, t), \\
B_{k} u(y, t) & =b_{k}\left(y ; D_{y}, D_{t}\right) u(y, t) .
\end{aligned}
$$

THEOREM. There are topological vector spaces $X_{1}, X_{2}, \ldots, X_{p} \subset \mathscr{D}(\Omega)$, dense in $L^{2}(\Omega)$, such that the mixed problem

$$
\begin{aligned}
A u(y, t) & =f(y, t) & & \text { on } \bar{\Omega} \times[0, \infty), \\
D_{t}^{j-1} u(y, 0) & =g_{j}(y) & & \text { on } \bar{\Omega} \times(0), 1 \leqq j \leqq p, \\
B_{k} u(y, t) & =0 & & \text { on } \partial \Omega \times[0, \infty), 1 \leqq k \leqq r,
\end{aligned}
$$

has a unique classical solution $u$ for each $g_{j} \in X_{j}$ and each continuous $f:[0, \infty) \rightarrow X_{p}$.

This formulation can be considered as an abstract analogue of the results in [3], [5], that the Cauchy problem has a unique solution when the data are in certain Gevrey classes; in fact the $X_{j}$ come from an abstract Gevrey space for the operator arising by reduction to a system of first order in time.

\section{REFERENCES}

1. R. Beals, Semigroups and abstract Gevrey spaces, J. Functional Analysis. 10 (1972).

2. J. Chazarain, Problèmes de Cauchy abstraits et applications à quelques problèmes mixtes, J. Functional Analysis 7 (1971), 386-446.

3. J. Leray and Y. Ohya, Équations et systèmes non-linéaires, hyperboliques non-strictes, Math. Ann. 170 (1967), 167-205. MR 34 \# 7946.

4. S. Matsuura, On non-strict hyperbolicity, Proc. Internat. Conference on Functional Analysis and Related Topics (Tokyo, 1969), Univ. of Tokyo Press, Tokyo, 1970, pp. 171-176. MR 42 \# 2174.

5. Y. Ohya, Le problème de Cauchy pour les équations hyperboliques à caractéristiques multiples. J. Math. Soc. Japan 16 (1964), 268-286. MR 31 \#3693.

Department of Mathematics, University of Chicago, Chicago, Illinois 60637 